\title{
Nanoarcillas sintéticas para ser empleadas en la valorización de glicerol a carbonato de glicerilo
}

\section{Synthetic nano-clays to be used in the valorization of glycerol to glycerol carbonate}

Presentación: 6-7/10/2020

\section{Doctorando:}

\section{Dalma Sofía Argüello}

Centro de Investigación y Tecnología Química, Consejo Nacional de Investigaciones Científicas y Técnicas, Facultad Regional Córdoba, Universidad Tecnológica Nacional, Argentina.

darguello@frc.utn.edu.ar

Directora:

Mónica Crivello

Co-directora:

Nancy Bálsamo

\section{Resumen}

La producción de biodiesel se acompaña de un $10 \%$ en peso de glicerol como subproducto, por lo que resulta factible que pueda ser utilizado como materia prima renovable para favorecer económicamente el proceso. La conversión catalítica de glicerol hacia un producto de mayor valor agregado, como el carbonato de glicerilo, es una opción atractiva porque se lo considera como un sustituto verde de importantes compuestos derivados del petróleo. Además, presenta numerosas aplicaciones en la industria química, farmacéutica, cosmética, etc. Como alternativa sustentable para producirlo se propone la transesterificación entre el glicerol y un alquil carbonato empleando catalizadores sólidos como los óxidos metálicos mixtos derivados de nanoarcillas aniónicas sintéticas, en condiciones suaves de reacción y sin el empleo de solvente. Estos materiales presentan gran interés por la posibilidad de nano-diseño a medida, control de accesibilidad a sitios activos y muy bajo costo de síntesis. Se sintetizaron óxidos de $\mathrm{Mg}$ y Al con $\mathrm{Cu}$ incorporado en diferentes contenidos, por el método de coprecipitación, para ser empleados en la reacción de transesterificación entre glicerol y etilencarbonato. Los materiales fueron caracterizados fisicoquímicamente mediante DTP- $\mathrm{CO}_{2}$, FTIR y XPS. Los materiales sintetizados mostraron una elevada actividad para la producción selectiva de carbonato de glicerilo, atribuido a la fuerte basicidad de superficie combinada con la presencia de sitios ácidos. El catalizador más activo fue el óxido con un contenido de Cu del 25\% con un 91,9\% de conversión de glicerol y 99,2 \% de selectividad a carbonato de glicerilo.

Palabras clave: Glicerol, Carbonato de glicerol, Catálisis heterogénea, Óxidos mixtos.

\section{Abstract}

Biodiesel production is accompanied by $10 \mathrm{wt} \%$ of glycerol as a by-product. Thus, the use of glycerol as a renewable raw material could be an economical advantage to the process. The catalytic conversion of glycerol to a higher value-added product such as glyceryl carbonate is an attractive option. Glyceryl carbonate is considered a 
green substitute for important petroleum-derived compounds. In addition, it has numerous applications in the chemical, pharmaceutical and cosmetic industries. The transesterification reaction between glycerol and an alkyl carbonate is a sustainable alternative when solid catalysts such as mixed metal oxides derived from synthetic anionic nano-clays are evaluated, along with under mild reaction conditions and without solvent employment. The great interest in the use of these materials is due to the possibility of custom nano-design, accessibility control to active sites and very low cost synthesis. The $\mathrm{Mg}$ and $\mathrm{Al}$ oxides with $\mathrm{Cu}$ incorporated in different loads were synthesized by the coprecipitation method. The materials were characterized physicochemically by TPD-CO2, FTIR and XPS and evaluated in the reaction between glycerol and ethylene. All samples with $\mathrm{Cu}$ incorporated showed significant activity to selective glyceryl carbonate production. Those catalytic performances could be attributed to the strong basicity on the surface combined with the acid sites presence of the materials. The most active catalyst was oxide with $25 \% \mathrm{Cu}$ content with $91.9 \%$ glycerol conversion and $99.2 \%$ selectivity to glyceryl carbonate.

Keywords: Glycerol, Glycerol carbonate, Heterogeneous catalysis, Mixed oxides.

\section{Introducción}

La producción de biodiesel se considera más segura para el medioambiente debido a la reducción en las emisiones con respecto a la producción del diésel de petróleo, pero ésta se acompaña de un $10 \%$ en peso de glicerol como subproducto, que puede ser utilizado como materia prima renovable para favorecer económicamente el proceso. El aumento de la demanda de biodiesel continuará suministrando glicerol en abundancia, lo que ha vuelto indispensable la búsqueda de nuevas vías de valorización, de aplicación y es aún materia pendiente a nivel científico como industrial.

La conversión catalítica de glicerol se ha investigado hacia productos de mayor valor agregado, tales como monoglicéridos (Bálsamo y col., 2019), acroleína, ácido glicérico y carbonato de glicerilo (CG), entre otros (Mazarío et al., 2020). El CG es una opción muy atractiva debido a que se trata de una sustancia química relativamente nueva en el mercado y es considerada un sustituto verde de importantes compuestos derivados del petróleo. Debido a su baja toxicidad, índice de evaporación, inflamabilidad y a su capacidad de hidratación, se lo emplea en la industria química, cosmética, farmacéutica (Sonnati et al., 2013), etc. Algunos autores reportan la síntesis de CG a partir de glicerol y urea con liberación de gas de amoníaco empleando altas temperaturas (Wang et al., 2018), o utilizando catalizadores como hidrotalcita y compuestos tipo hidrotalcita (Marimuthu et al., 2018). Sin embargo, el uso de urea requiere trabajar a presión reducida con el fin de separar el gas amoníaco y evitar la formación de productos secundarios indeseables. Otro método utilizado para obtener el CG es la transesterificación entre glicerol y fosgeno, compuesto tóxico. En contraposición, la alternativa más prometedora es la transesterificación entre glicerol y alquil carbonatos, debido a que las condiciones del proceso son más favorables con el medio ambiente (Climent et al., 2010). Recientemente se han empleado óxidos mixtos de Al-Mg dopado con diferentes cationes logrando una importante actividad cuando se incorporó Ni.

Entre los materiales viables para ser utilizados como catalizadores heterogéneos, se encuentran los óxidos metálicos mixtos (OMM) obtenidos por descomposición térmica de los hidróxidos dobles laminares (HDL). Los HDL son un tipo de nanoarcillas aniónicas de estructura laminar; cuando su composición es solo $\mathrm{Mg}$ y Al, se los denomina hidrotalcita. La fórmula general $\left[\mathrm{M}^{2+}(1-\mathrm{x}) \mathrm{M}^{3+}{ }_{\mathrm{x}}(\mathrm{OH})_{2}\right]^{\mathrm{x}+}\left[\mathrm{A}_{\mathrm{x} / \mathrm{n}}^{\mathrm{n}} \mathrm{mH}_{2} \mathrm{O}\right]^{\mathrm{x}-}$, donde $\mathrm{M}^{2+} \mathrm{y} \mathrm{M}^{3+}$ son cationes de metales, di y trivalentes $\mathrm{Mg}, \mathrm{Cu}, \mathrm{Fe}, \mathrm{Co}, \mathrm{Al}, \mathrm{Zn}$, etc., aunque también se pueden formar este tipo de estructuras empleando metales mono y tetravalentes, como: Li, Cs, La, Ce, etc., mientras que A representa el anión compensador de cargas, generalmente carbonato, que junto con " $m$ " moléculas de agua se ubican en el espacio interlaminar; $x$ puede variar entre 0,17 y 0,33, dependiendo de la combinación de metales di y trivalentes. Las propiedades de los HDL están fuertemente influenciadas por la composición y naturaleza de los aniones y cationes. La incorporación de un tercer metal en la estructura de los HDL permite modificar su comportamiento catalítico tanto en la actividad como en la selectividad. Los OMM alcanzan una composición homogénea a escala molecular y muestran un comportamiento particular comparado con los óxidos metálicos puros, debido a la combinación de distintas fases en la red molecular, presentan alta área superficial, estabilidad térmica y dispersión homogénea de la fase activa. De allí la importancia de diseñar un material con propiedades específicas tanto en composición como características ácidas, básicas o redox que favorezcan su utilización como catalizadores en las reacciones de transesterificación. En este trabajo se 
estudió la conversión catalítica de glicerol y etilencarbonato a CG utilizando OMM con distinto contenido de cobre, como catalizadores.

\section{Desarrollo}

Los HDL sintetizados por el método de coprecipitación se calcinaron a $450^{\circ} \mathrm{C}$ por $9 \mathrm{~h}$ para obtener los $\mathrm{OMM}$ (Argüello, 2019). Los porcentajes molares de Cu incorporado fueron de 15, 20 y 25\% con respecto a los moles de $\mathrm{Mg}$ en el material, manteniendo una relación catiónica molar, $\left(\mathrm{Mg}^{2+}+\mathrm{Cu}^{2+}\right): \mathrm{Al}^{3+}$, constante e igual a 3. Los materiales se denominaron según la composición de los metales y el contenido de $\mathrm{Cu}$ incorporado, por ejemplo: $\mathrm{Cu}_{25} \mathrm{MgAl}$.

En cuanto a la caracterización de los materiales, complementando los estudios realizados sobre estructura, composición y área superficial (Argüello, 2019), se utilizó desorción a temperatura programada con molécula sonda de $\mathrm{CO}_{2}$ (DTP) para determinar tipo y densidad específica de sitios básicos existentes en la superficie de los OMM. Mediante espectroscopía fotoelectrónica de rayos X (XPS) se determinó el estado químico y la estructura electrónica de los elementos de los materiales. Se realizó espectroscopía de infrarrojo con Transformada de Fourier (FTIR) a los efectos de identificar los posibles sitios ácidos de los materiales, para tal fin se utilizó la molécula sonda de piridina.

La síntesis de CG a partir de glicerol (Gly) y etilencarbonato (EC), representada en la Figura 1, se realizó en un reactor batch de vidrio equipado con un agitador magnético y un condensador de reflujo. Se utilizó una relación molar 2/1 de EC/Gly. Las reacciones se realizaron a $45^{\circ} \mathrm{C}$ y a $70{ }^{\circ} \mathrm{C}$ en ausencia de disolvente y con atmósfera de nitrógeno. Se utilizó $2,55 \%$ en peso de catalizador con respecto a la masa total de reactivos. Los tiempos de reacción evaluados fueron: 45,60 y 90 minutos a $45{ }^{\circ} \mathrm{C}$; 30, 60 y $90 \mathrm{~min}$ a $70{ }^{\circ} \mathrm{C}$. Se compararon los catalizadores OMM con distinto contenido de cobre con respecto al óxido de MgAl sin cobre incorporado. Después del tiempo de reacción, se separó el catalizador sólido mediante centrifugación del medio de reacción y se agregó acetona al líquido sobrenadante para analizarlo por cromatografía gaseosa con detector de ionización a la llama. Para los cálculos de conversión, rendimiento y selectividad se usó el método de normalización de área empleando factores de respuesta.

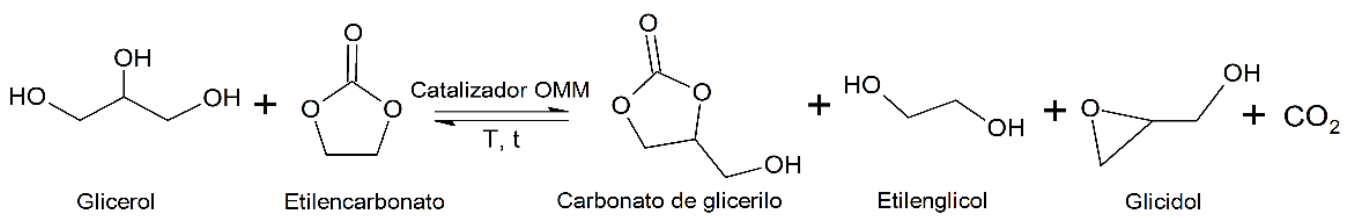

Figura 1. Reacción de transesterificación.

La Figura 2 muestra las curvas de desorción de $\mathrm{CO}_{2}$ a distintas temperaturas con el fin de determinar tipo y densidad específica de sitios básicos de la superficie de los OMM, ya que la basicidad del catalizador juega un papel importante en la transesterificación. La distribución de la fuerza básica se calculó para todas las muestras y se detalla en la Tabla 1. Los sitios básicos de Brönsted (débiles) y Lewis (fuertes) son producidos por la presencia de los grupos $\mathrm{OH}^{-}$y los iones $\mathrm{O}^{2-}$ respectivamente. Los sitios débilmente básicos $\left(50-250{ }^{\circ} \mathrm{C}\right)$ correspondían a grupos $\mathrm{OH}^{-}$, los sitios moderadamente básicos $\left(250-450^{\circ} \mathrm{C}\right)$ se asignaron a pares de metal-oxígeno, y sitios fuertemente básicos $\left(>450{ }^{\circ} \mathrm{C}\right)$ se derivaron de átomos de oxígeno de baja coordinación $\left(\mathrm{O}^{2-}\right)$ (Wang et al., 2018). En todas las muestras de OMM con $\mathrm{Cu}$ se observó un pico de desorción a alta temperatura $\left(835^{\circ} \mathrm{C}\right)$, el mismo se lo puede atribuir a la generación de sitios básicos muy fuertes. Además, la fuerte basicidad podría atribuirse a la presencia de una cantidad óptima de especies de Cu en la superficie del OMM-MgAl que produce sitios básicos de Lewis fuertes en gran cantidad. Estos volúmenes de adsorción de $\mathrm{CO}_{2}$ son más altos que los OMM reportados por Marimuthu, debido principalmente, a las mayores áreas superficiales (Argüello, 2019) ya que el contenido de Cu es semejante.

Con el análisis de XPS se obtuvo más información sobre la naturaleza química de las especies de Cu de la superficie. En la Figura 3 se muestran las regiones de $\mathrm{Mg} 1 \mathrm{~s}, \mathrm{Cu} 2 \mathrm{p}$ y $\mathrm{O}$ 1s del $\mathrm{OMM}-\mathrm{Cu}_{20} \mathrm{MgAl}$ después de la deconvolución. El pico de $\mathrm{Mg}$ 1s se ubica a 1302,2 eV, al deconvolucionarlo se observan dos contribuciones a 1301,6 eV y 1304 eV que podrían atribuirse a $\mathrm{Mg}(\mathrm{OH})_{2}$ y MgO, respectivamente (Marimuthu et al., 2018), siendo mayor la presencia del MgO. La región del $\mathrm{Cu} 2 \mathrm{p}$ muestra el pico predominante del $\mathrm{Cu} 2 \mathrm{p}_{3 / 2}$ a 932,8 eV y su correspondiente pico satélite shake-up a 941,5 eV, de acuerdo con los informes de la literatura. Esto indicaría la presencia principal 
del estado de oxidación $\mathrm{Cu}^{2+}$ en la muestra. La región del O 1s muestra dos contribuciones a 531,5 eV y 530,41 eV que corresponderían a $\mathrm{OH}^{-}$y $\mathrm{O}^{2-}$ de superficie, respectivamente. La disponibilidad de las especies $\mathrm{OH}^{-}$y $\mathrm{O}^{2-}$ de la muestra de OMM, $\mathrm{Cu}_{20} \mathrm{MgAl}$, analizada por XPS serían responsables de la basicidad de la superficie que se reporta precedentemente por DTP.

\begin{tabular}{|c|c|c|c|c|c|}
\hline \multirow{2}{*}{ OMM } & \multicolumn{5}{|c|}{ Densidad de sitios básicos $\left(\mathrm{mmol}^{-1}\right)$} \\
\cline { 2 - 6 } & $\begin{array}{c}\text { Débiles } \\
\sim 50-250^{\circ} \mathrm{C}\end{array}$ & $\begin{array}{c}\text { Medios } \\
\sim 250-450^{\circ} \mathrm{C}\end{array}$ & $\begin{array}{c}\text { Fuertes } \\
\sim 450-750^{\circ} \mathrm{C}\end{array}$ & $\begin{array}{c}\text { Muy fuertes } \\
>750^{\circ} \mathrm{C}\end{array}$ & Totales \\
\hline $\mathrm{MgAl}$ & 1,13 & 0,46 & 0,63 & - & 2,21 \\
\hline $\mathrm{Cu}_{15} \mathrm{MgAl}$ & 0,77 & 0,49 & 0,42 & 0,16 & 1,78 \\
\hline $\mathrm{Cu}_{20} \mathrm{MgAl}$ & 1,10 & 0,47 & 0,27 & 0,34 & 2,16 \\
\hline $\mathrm{Cu}_{25} \mathrm{MgAl}$ & 0,54 & 0,41 & 0,11 & 0,26 & 1,32 \\
\hline
\end{tabular}

Tabla 1. Propiedades básicas de los catalizadores.

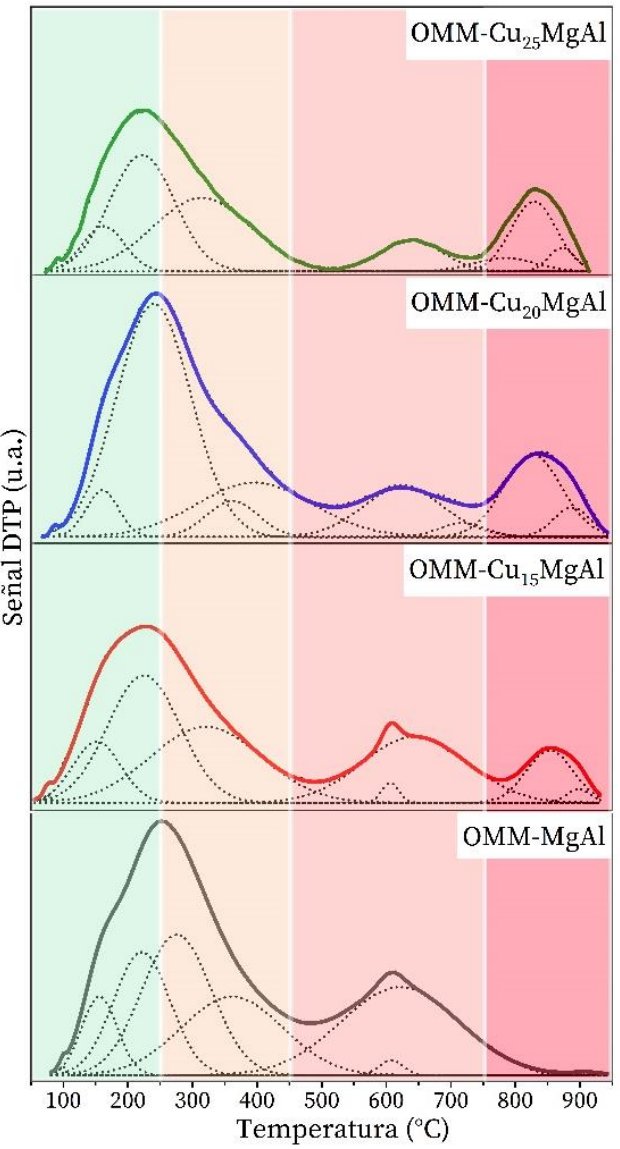

Figura 2. Perfiles de DTP- $\mathrm{CO}_{2}$ de los OMM.
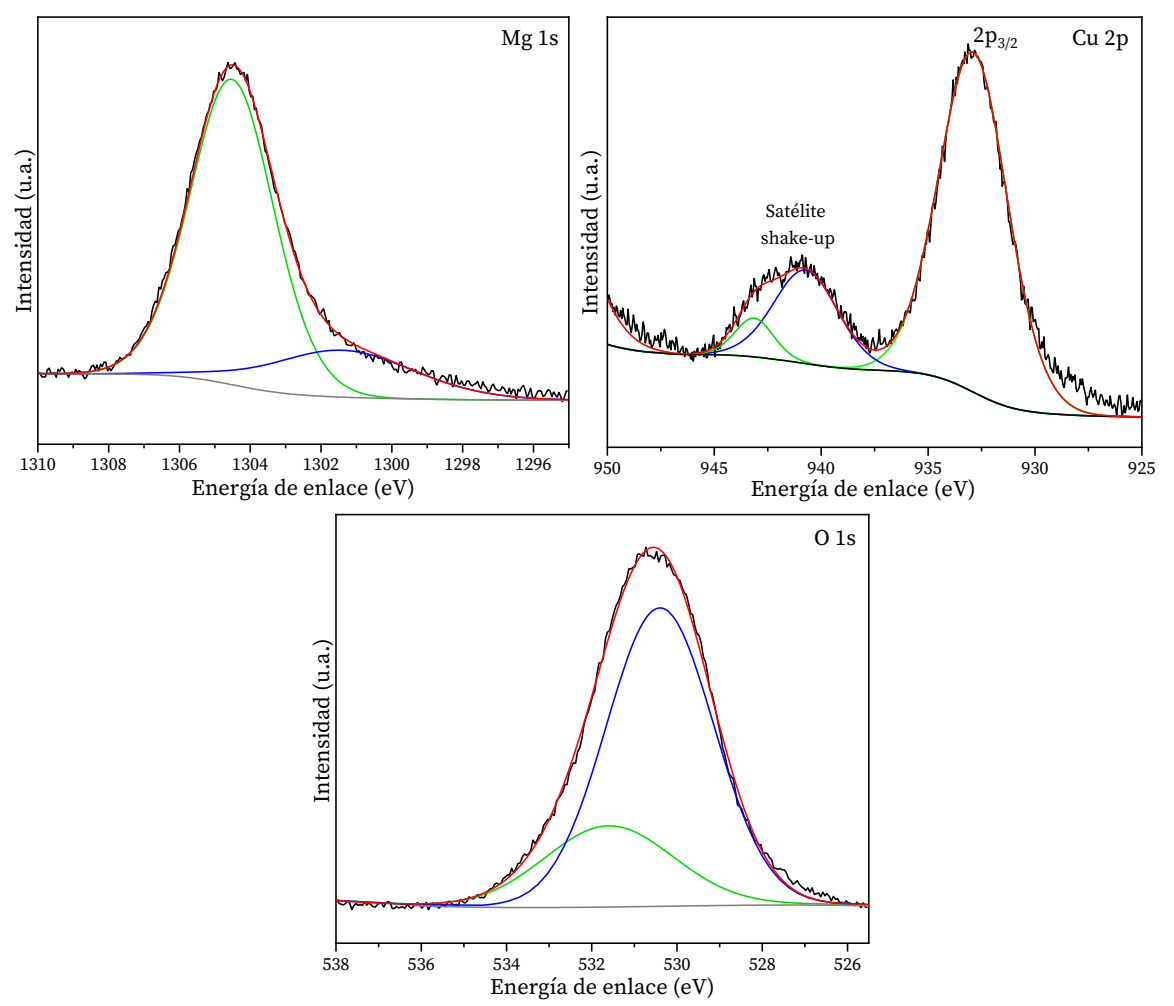

Figura 3. Espectros de XPS del OMM-Cu${ }_{20} \mathrm{MgAl}$.

Los espectros (FTIR) medidos después de la adsorción y evacuación de piridina a $50{ }^{\circ} \mathrm{C}$ en los $\mathrm{OMM}$ de $\mathrm{MgAl}$, $\mathrm{Cu}_{20} \mathrm{MgAl}$ y $\mathrm{Cu}_{25} \mathrm{MgAl}$ se muestran en la Figura 4. Las vibraciones de estiramiento de moléculas de piridina unidas coordinadamente a los sitios ácidos de Lewis están entre 1630 y $1600 \mathrm{~cm}^{-1}$ (modo v8a) y 1450 y $1440 \mathrm{~cm}^{-1}$ (modo v19b) (Prescott et al., 2005). El número de onda del modo v8a es sensible a la fuerza de la acidez de Lewis, mientras que la intensidad del modo v19b caracteriza el número de dichos sitios. No se encontraron sitios ácidos de Brönsted (a 1640 y $1540 \mathrm{~cm}^{-1}$ ) en las muestras de $\mathrm{OMM}$ de $\mathrm{MgAl}$ y Cu $\mathrm{Cu}_{20} \mathrm{MgAl}$, excepto una banda a $1540 \mathrm{~cm}^{-1}$ en el OMM-Cu${ }_{25} \mathrm{MgAl}$. Con

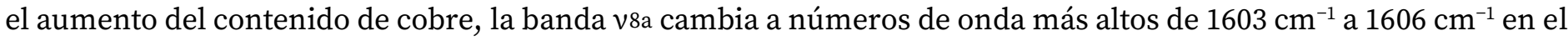
$\mathrm{OMM}-\mathrm{Cu}_{25} \mathrm{MgAl}$ (Fig. 4), lo que corresponde a un aumento en la fuerza del sitio ácido y también se observa un 
incremento en el número de dichos sitios. Coincidiendo con lo planteado por Ji y col. es factible que la actividad catalítica de los óxidos mixtos pueda atribuirse a una combinación de sitios básicos y ácidos superficiales (Ji, 2019).

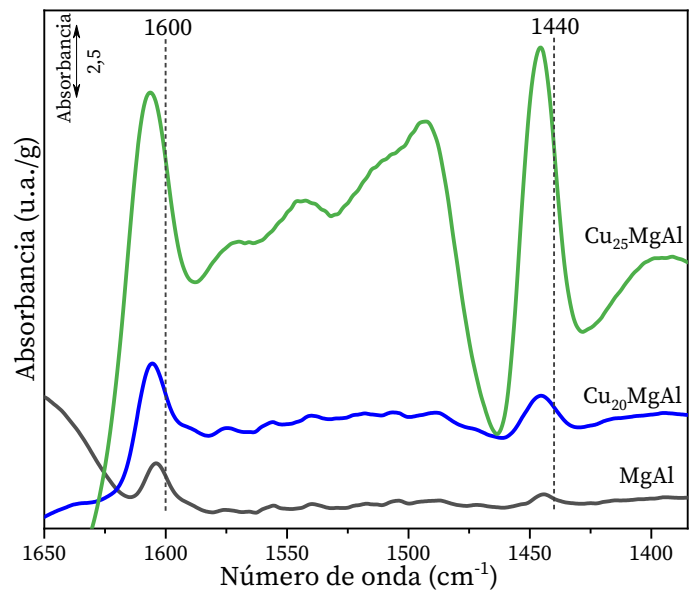

Figura 4. Adsorción de piridina por FTIR en los OMM. Espectro obtenido después de la adsorción y evacuación a $50^{\circ} \mathrm{C}$.

Durante la evaluación catalítica a ambas temperaturas $\left(45\right.$ y $\left.70{ }^{\circ} \mathrm{C}\right)$, todos los catalizadores mostraron resultados significativos. Con el fin de disminuir la formación de glicidol, se comenzó probando los materiales a la temperatura de reacción de $45^{\circ} \mathrm{C}$ y se obtuvieron valores de conversión de glicerol de 55,2 a 94,5\%, de selectividad a CG de 63,9 a $98,6 \%$ y a GD de 1,4 a 36,1\% para los distintos tiempos evaluados. Los rendimientos a CG se muestran en la Figura 5. Bajo estas condiciones de reacción, el mayor rendimiento a CG se observó a los 90 minutos con el OMM Cu${ }_{15} \mathrm{MgAl}$. A la temperatura de reacción de $70{ }^{\circ} \mathrm{C}$, los valores de conversión de glicerol estuvieron en el rango de 87,4 a 96,7\% y de selectividad a carbonato de glicerol de 51,4 a 99,2\% para los diferentes tiempos evaluados. Los rendimientos a CG se grafican en la Figura 6. Se produjo glicidol (GD) como un subproducto (0,8-48,6\%), debido a la descarbonilación del CG, tal como ha sido reportado por otros autores, sobre catalizadores con elevada fuerza básica. En la Tabla 2 se muestran los parámetros catalíticos a $70{ }^{\circ} \mathrm{C}$ y $30 \mathrm{~min}$ de reacción.

\begin{tabular}{|c|c|c|c|c|}
\hline \multirow{2}{*}{ OMM } & \multirow{2}{*}{ Conversión Gly (\%) } & \multicolumn{2}{|c|}{ Selectividad (\%) } & \multirow{2}{*}{ Rendimiento CG (\%) } \\
\cline { 3 - 4 } & & CG & GD & \\
\hline $\mathrm{MgAl}$ & 91,7 & 94,1 & 5,9 & 86,3 \\
\hline $\mathrm{Cu}_{15} \mathrm{MgAl}$ & 93,3 & 76,6 & 23,4 & 71,5 \\
\hline $\mathrm{Cu}_{20} \mathrm{MgAl}$ & 94,3 & 54,0 & 46,0 & 50,9 \\
\hline $\mathrm{Cu}_{25} \mathrm{MgAl}$ & 91,9 & 99,2 & 0,8 & 91,2 \\
\hline
\end{tabular}

Tabla 2. Conversión, selectividad y rendimiento.

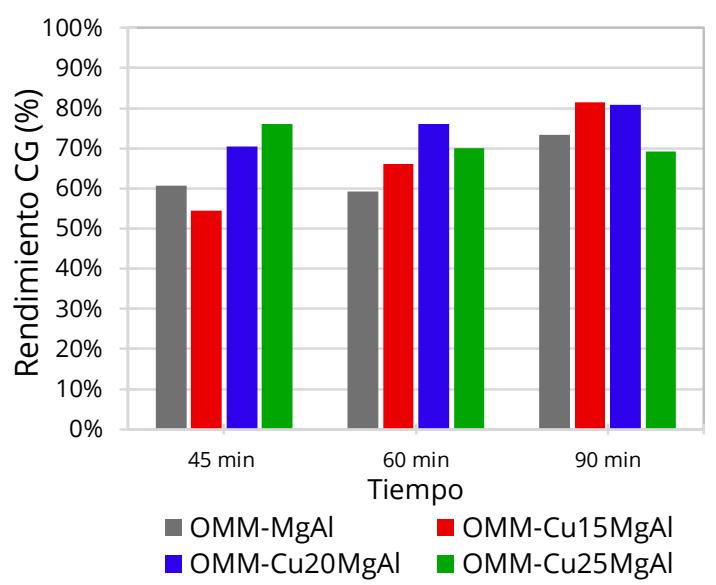

Figura 5. Rendimiento a CG de los catalizadores OMM en la reacción de transesterificación a $45^{\circ} \mathrm{C}$.

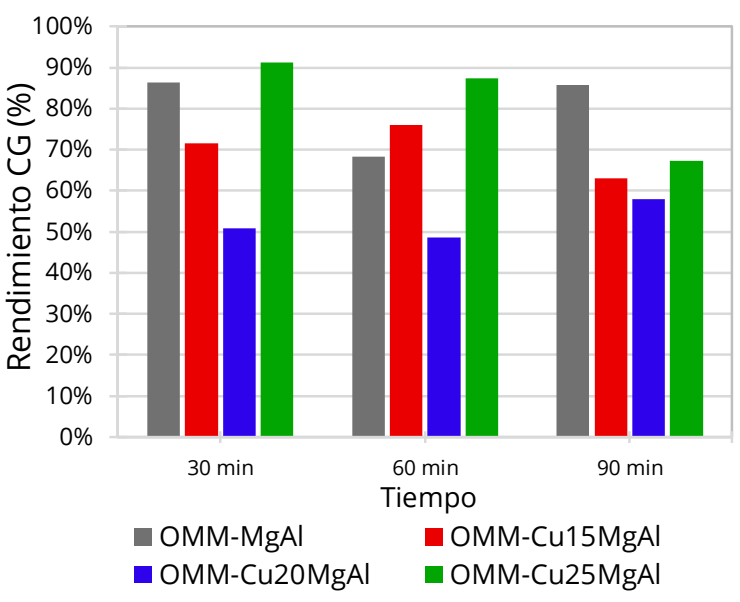

Figura 6. Rendimiento a CG de los catalizadores OMM en la reacción de transesterificación a $70^{\circ} \mathrm{C}$. 
Bajo estas condiciones de reacción, el OMM- $\mathrm{Cu}_{25} \mathrm{MgAl}$ presentó el mayor rendimiento a CG a los 30 minutos junto con la menor formación de glicidol. Dicho comportamiento podría atribuirse a la combinación adecuada de sitios básicos y ácidos presentes en superficie. La literatura informada enfatizó que la reacción de transesterificación se ve facilitada por la disponibilidad de sitios de una fuerte basicidad de Lewis (Marimuthu et al., 2018). Los resultados actuales apuntan a que los sitios básicos fuertes de los OMM de $\mathrm{Cu}$ juegan un papel importante en la adquisición de un alto rendimiento de CG. No obstante, un mecanismo de reacción hipotético para la transesterificación de glicerol con EC podría ser promovido mediante dos modos de activación, que involucran sitios básicos y ácidos (Ji, 2019).

\section{Conclusiones}

Todos los materiales presentaron propiedades básicas. La basicidad de los OMM, con distintas cargas de $\mathrm{Cu}$ incorporado, se incrementó en densidad de sitios muy fuertes con el aumento del contenido de $\mathrm{Cu}$. El OMM de $\mathrm{Cu}_{25} \mathrm{MgAl}$ exhibió sitios ácidos de Lewis en mayor cantidad y fortaleza con respecto a los OMM de $\mathrm{MgAl}$ y Cu${ }_{20} \mathrm{MgAl}_{\text {. }}$ Aún falta determinar la acidez en el $\mathrm{OMM}-\mathrm{Cu}_{15} \mathrm{MgAl}$ para lograr una óptima comparación. La composición superficial del OMM-Cu ${ }_{20} \mathrm{MgAl}$ analizada por XPS se correlacionó en gran medida con lo reportado previamente en el análisis de fases por DRX. En cuanto a la evaluación catalítica, el $\mathrm{OMM}-\mathrm{Cu}_{25} \mathrm{MgAl}$ presentó el mayor rendimiento a CG $(91,2 \%)$ a los 30 minutos de reacción y a una temperatura de $70^{\circ} \mathrm{C}$. Estos resultados se podrían atribuir a una adecuada distribución de la basicidad y acidez de superficie.

\section{Referencias}

Argüello, D. (2019) Nanoarcillas sintéticas para ser empleadas en la valorización de glicerol a carbonato de glicerilo. Jornada de Intercambio y Difusión de los Resultados de Investigaciones de los Doctorados en Ingeniería. https://doi.org/10.33414/ajea.4.424.2019.

Bálsamo, N., Mendieta, S., Heredia, A., \& Crivello, M. (2019). Nanoclays as dispersing precursors of La and Ce oxide catalysts to produce high-valued derivatives of biodiesel by-product. Molecular Catalysis, 0-1. https://doi.org/10.1016/j.mcat.2019.01.010.

Climent, M. J., Corma, A., De Frutos, P., Iborra y otros. (2010). Chemicals from biomass: Synthesis of glycerol carbonate by transesterification and carbonylation with urea with hydrotalcite catalysts. The role of acid-base pairs. Journal of Catalysis, 269(1), 140-149. https://doi.org/10.1016/j.jcat.2009.11.001.

Ji. (2019). Recent Development of Heterogeneous Catalysis in the Transesterification of Glycerol to Glycerol Carbonate. Catalysts, 9(7), 581. https://doi.org/10.3390/catal9070581.

Marimuthu, M., Marimuthu, P., Ashok, A. K., Palanivelu, S., \& Rajagopalan, V. (2018). Tuning the basicity of Cubased mixed oxide catalysts towards the efficient conversion of glycerol to glycerol carbonate. Molecular Catalysis, 460, 53-62. https://doi.org/10.1016/j.mcat.2018.09.002.

Mazarío, J., Concepción, P., Ventura, M., \& Domine, M. E. (2020). Continuous catalytic process for the selective dehydration of glycerol over Cu-based mixed oxide. Journal of Catalysis, 385, 160-175. https://doi.org/10.1016/j.jcat.2020.03.010.

Prescott, H. A., Li, Z. J., Kemnitz, E., Trunschke, A. y otros. (2005). Application of calcined Mg-Al hydrotalcites for Michael additions: An investigation of catalytic activity and acid-base properties. Journal of Catalysis, 234(1), 119130. https://doi.org/10.1016/j.jcat.2005.06.004.

Sonnati, M. O., Amigoni, S., Taffin, E. P., Darmanin, T. y otros. (2013). Glycerol carbonate as a versatile building block for tomorrow: Synthesis, reactivity, properties and applications. Green Chemistry, 15(2), 283-306. https://doi.org/10.1039/c2gc36525a.

Wang, D., Zhang, X., Cong, X., Liu, S., \& Zhou, D. (2018). Influence of Zr on the performance of Mg-Al catalysts via hydrotalcite-like precursors for the synthesis of glycerol carbonate from urea and glycerol. Applied Catalysis A: General, 555, 36-46. https://doi.org/10.1016/j.apcata.2018.02.009. 